\title{
The anti-obesity effect of lotus leaves on high-fat-diet-induced obesity by modulating lipid metabolism in C57BL/6J mice
}

\author{
Ya Wu $u^{1,2,3,4}$, Fang Tan ${ }^{5}$, Tianyu Zhang ${ }^{1,4}$, Binglin Xie ${ }^{1,4}$, Lixian Ran ${ }^{1,4}$ and Xin Zhao ${ }^{1,2,3^{*}}$ (D)
}

\begin{abstract}
Lotus leaves (Nelumbo nucifera) are widely used in medicines and foods. The investigate systematically studied the anti-obesity effect of lotus leaf extracts. It could reduce body weight, alleviate liver damage, and inhibit fat accumulation in high-fat-diet-induced obese mice. Lotus leaf extracts reduced serum alanine aminotransferase (ALT), aspartate transaminase (AST), and alkaline phosphatase (AKP) levels; decreased total cholesterol (TC), triglycerides (TG), and low-density lipoprotein cholesterol (LDL-C) levels in the serum; and increased high-density lipoprotein cholesterol (HDL-C) levels to improve dyslipidemia. Lotus leaves also inhibited inflammation accompanied by obesity via decreasing inflammatory cytokine interleukin (IL)-1 $\beta$, tumor necrosis factor-a (TNF-a), interferon gamma (IFN- $\gamma$ ), and IL-6 levels and increasing anti-inflammatory cytokine IL-4 and IL-10 levels. QPCR analysis revealed that lotus leaves upregulated peroxisome proliferator-activated receptor alpha (PPAR-a), lipoprotein lipase (LPL), carnitine palmitoyltransferase 1 (CPT1), and cholesterol 7 alpha hydroxylase (CYP7A1) mRNA expressions and downregulated peroxisome proliferator-activated receptor gamma (PPAR- $\gamma$ ) and CCAAT/enhancer-binding protein alpha (C/EBP-a) mRNA expressions, to reduce adipocyte differentiation and fat accumulation, promote oxidation of fat and decomposition of triglyceride and cholesterol. So, lotus leaves effectively regulated lipid metabolism, alleviated inflammation and liver injury in obese mice; thus, lotus leaves could be further developed as a food to combat obesity.
\end{abstract}

Keywords: Lotus leaves, High-fat diet, Anti-obesity, Lipid metabolism, Anti-inflammation, Liver injury

\section{Introduction}

Obesity is a metabolic disorder caused by excessive fat accumulation, which results in abnormal weight gain [1]. The obese population is growing rapidly, affecting younger populations and becoming more difficult to control [2], as well as a global epidemic. Obesity is closely related to the occurrence of various chronic metabolic diseases such as nonalcoholic fatty liver disease [3], cardiovascular disease [4], and type 2 diabetes [5], which seriously affect the quality of life of patients. Obesity also contributes to a variety of cancers in humans by

\footnotetext{
*Correspondence: zhaoxin@cque.edu.cn

${ }^{1}$ Chongqing Collaborative Innovation Center for Functional Food,

Chongqing University of Education, Chongqing 400067, China

Full list of author information is available at the end of the article
}

damaging the immune system [6]. Although current obesity treatment drugs have certain weight loss effects, the adverse effects are relatively large [7]. Therefore, development of safe, efficient, natural anti-obesity medicines and functional foods has become a research focus in the health industry.

Lotus leaves are fresh or dried leaves of the perennial aquatic plant Nelumbo nucifera [8], which is both medicinal and edible. Lotus leaves have a long history of being planted and used in China and are widely distributed and cultivated in drainage basins of the Yellow, Changjiang and Zhujiang Rivers in China [9]. The main active constituents in lotus leaves are flavonoids and alkaloids. Studies have shown that lotus leaf flavonoids mainly include quercetin, isoquercetin, kaempferol and other monomers or aglycones, and the principal alkaloids are 
monobenzyl isoquinoline, bisbenzyl isoquinoline, apomorphine, dehydroaporphine, oxidized apomorphine, and proaporphine [10]. The studies have shown that lotus leaf extracts have extensive pharmacological activities, including anti-obesity [11], antioxidation [12], anti-cardiovascular disease [13], and anti-cancer [14].

According to the researches on the anti-obesity effect of lotus leaves, it is found that most of the experiments in vitro introduced the extract or the isolated monomer compounds are useful for the suppression of obesity in adipocyte by inhibiting lipase activity, lipogenesis and adipocyte differentiation [15-18]. In vivo experiments, the anti-obesity effects were measured by body weight, blood lipids and liver function indicators in obese mice induced by high-fat diet [19-21]. However, obesity, as a chronic metabolic disease, affects systemic functions. It not only causes dyslipidemia, liver damage, but also leads to low-grade inflammation, which further promote the development of physiological disorders such as insulin resistance and metabolic syndrome [22]. In 2018, Kim et al. reported that the lotus leaf water extract improved the antioxidant capacity of obese mice and reduced the level of oxidative stress, but they did not clarify the mechanism of action [23]. Back in 2010, Wu et al. reported that lotus leaf water extracts reduced lipid accumulation by increasing the phosphorylation of AMP-activated protein kinase, and inhibiting the expression of acetyl-CoA carboxylase, fatty acid synthetase (FAS) and 3-hydroxy3-methyl glutaryl coenzyme A reductase [24].

Lotus leaves have the advantages of being abundant and cheap, with various physiological activities. The present study focused on obesity as a systemic disease, and not only detected classic blood lipids and liver function indicators (TC, TG, LDL-C, HDL-C, ALT, AST, AKP). At the same time, the degree of chronic low-grade inflammation accompanied by obesity, were measured by inflammatory factors, were measured by antioxidant levels and inflammatory factors. In addition, unlike previous Wu's reports that focused on the mechanism of lipid synthesis, we started from the PPAR pathway to detect the expression of related genes, and explored anti-obesity mechanism of lotus leaf extracts. The results may provide ideas for dietary therapy and interventions for the growing obese population.

\section{Materials and methods}

\section{Preparation of lotus leaf extract}

Lotus leaves (20 g; Anhui Provinces) were crushed, and $70 \%$ ethanol was added at a liquid/material ratio of 20:1. After heating at $60^{\circ} \mathrm{C}$ for $3 \mathrm{~h}$, the mixed liquid was cooled and filtered to obtain the crude extract, which was then purified via FL-3 macroporous absorbent column chromatography [25] (Shanghai Lanji Technology
Development Co., Ltd., Shanghai, China). Ethanol (70\%) was used as eluant to flush the column until the eluant became colorless. The eluted solution was collected and evaporated, and the residue was dried and ground to obtain the lotus leaf extract.

\section{Determination of lotus leaf extract composition}

Two milligrams each of hyperoside (Beijing Solibao Technology Co., Ltd., Beijing, China), kaempferitrin, astragalin, phlorizin, and quercetin (Beijing Putian Tongchuang Biological Technology Co., Ltd., Beijing, China) were accurately weighed, then dissolved in $2 \mathrm{~mL}$ of methanol (HPLC grade) to prepare the standard solution. Lotus leaf extract $(10 \mathrm{mg})$ was dissolved in $1 \mathrm{~mL}$ of DMSO, then $1.5 \mathrm{~mL}$ methanol and $1.5 \mathrm{~mL} \mathrm{H}_{2} \mathrm{O}$ were added. Samples were filtered through a $0.22-\mu \mathrm{m}$ microporous membrane to obtain the test solution.

Separation chromatography was performed using an UltiMate3000 HPLC System (Thermo Fisher Scientific, Waltham, MA, USA) and a Thermo Accucore C18 column $(150 \times 1.6 \mathrm{~mm}, 2.6 \mu \mathrm{m})$. Mobile phase A was acetonitrile (HPLC grade); mobile phase B was $0.5 \%$ glacial acetic acid aqueous solution. The mobile phase gradient was: $0 \mathrm{~min}, 12 \% \mathrm{~A} ; 0-30 \mathrm{~min} 40 \% \mathrm{~A} ; 30-35 \mathrm{~min} 100 \%$ A; $35-40 \mathrm{~min}, 100 \% \mathrm{~A}$. The flow rate was $0.5 \mathrm{~mL} / \mathrm{min}$; the column temperature was $35{ }^{\circ} \mathrm{C}$; the detection wavelength was $360 \mathrm{~nm}$; and the injection volume was $10 \mu \mathrm{L}$. Flavonoid types were determined according to retention time and analyzed using the chromatographic peak area of each component.

\section{Animal models and treatment}

Fifty healthy C57BL/6J mice (weighing $20 \pm 2$ g, 25 males and 25 females) were randomly divided into the normal, model, L-carnitine, low-concentration lotus leaf extract (LL-L), and high-concentration lotus leaf extract (LL-H) groups. The normal group received normal maintenance foods and drinking water; the other groups received a D1249251 high-fat diet (Chongqing Medical University, Chongqing, China) and 10\% sugar water. After 2 weeks, without changing the diet, mice in the L-carnitine group were intragastrically administered $200 \mathrm{mg} / \mathrm{kg}$ L-carnitine daily, mice in the LL-L group were intragastrically administered $100 \mathrm{mg} / \mathrm{kg}$ lotus leaf extract daily, and mice in the LL-H group were intragastrically administered $200 \mathrm{mg} /$ $\mathrm{kg}$ lotus leaf extract daily [26]. After 7 weeks, all mice were fasted for $12 \mathrm{~h}$ and humanely killed. Blood was collected from the orbits, and the epididymal fat (from the males) and livers were collected for further experiments. The liver and epididymal fat indexes were calculated as organ weight $(\mathrm{g}) /$ mouse weight $(\mathrm{g}) \times 100$. 


\section{Detection of serum AKP, AST, ALT, TC, TG, LDL-C and HDL-C levels}

The plasma was centrifuged at $4000 \mathrm{rpm}$ for $10 \mathrm{~min}$, then the supernatant was collected. Serum alanine aminotransferase (ALT), aspartate transaminase (AST), alkaline phosphatase (AKP) levels, total cholesterol (TC), triglyceride (TG), high-density lipoprotein cholesterol (HDL-C), and low-density lipoprotein cholesterol (LDLC) levels were measured using kits (Nanjing Jiancheng Bioengineering Institute, Nanjing, China).

\section{Determination of serum cytokine TNF- $\alpha$, IFN- $\gamma$, IL- 6 , IL-1 $\beta$,} IL-4, and IL-10 levels

The mouse blood was centrifuged at $4000 \mathrm{rpm}$ for $10 \mathrm{~min}$, then the supernatant was carefully collected. The serum cytokine levels of tumor necrosis factor-alpha (TNF- $\alpha$ ), interferon gamma (IFN- $\gamma$ ), and interleukin (IL) $-1 \beta$, IL-6, IL-4, and IL-10 were measured according to each kit's instructions (Beijing Chenglin Biotechnology Co., Ltd., Beijing, China).

\section{Pathological examination of the liver and epididymal fat}

Liver and epididymal fat $\left(\sim 0.5 \mathrm{~cm}^{2}\right)$ were fixed in $10 \%$ formalin for $48 \mathrm{~h}$, then processed, dehydrated, embedded in paraffin, sectioned, and stained with hematoxylin and eosin. Tissue histology was examined under an optical microscope (BX43 microscope, Olympus, Tokyo, Japan).

\section{Quantitative PCR (qPCR) assay}

The mouse liver tissues were pulverized; total RNA (Thermo Fisher Scientific, Inc., Waltham, MA, USA) was extracted with RNAzol reagent (Invitrogen, Carlsbad, CA, USA), and the concentration of the extracted total RNA was diluted to $1 \mu \mathrm{g} / \mu \mathrm{L}$. Next, $5 \mu \mathrm{L}$ of diluted total RNA solution was used for reverse transcription to synthesize the cDNA template as per the kit instructions (Tiangen Biotech Co., Ltd., Beijing, China). The cDNA template $(1 \mu \mathrm{L})$ was mixed with $10 \mu \mathrm{L}$ SYBR Green PCR Master Mix and $1 \mu \mathrm{L}$ upstream and downstream primers (Table 1). The solution was reacted at $95^{\circ} \mathrm{C}$ for $30 \mathrm{~s}$, followed by 40 cycles of $95^{\circ} \mathrm{C}$ for $30 \mathrm{~s}, 55^{\circ} \mathrm{C}$ for $30 \mathrm{~s}, 72{ }^{\circ} \mathrm{C}$ for $30 \mathrm{~s}$, and a final extension at $95{ }^{\circ} \mathrm{C}$ for $30 \mathrm{~s}$, followed by $55{ }^{\circ} \mathrm{C}$ for $35 \mathrm{~s}$. The relative gene expressions were calculated via the $2^{-\Delta \Delta C t}$ method. Glyceraldehyde-3-phosphate dehydrogenase (GAPDH) served as the internal reference.

\section{Statistical analysis}

The data were analyzed using SPSS 17.0 and GraphPad Prism 7 statistical software. Experimental results are expressed as the mean \pm standard deviation. One-way
Table 1 Sequences of primers used in this study

\begin{tabular}{|c|c|}
\hline Gene name & Sequence \\
\hline$L P L$ & $\begin{array}{l}\text { Forward: 5'-AGGGCTCTGCCTGAGTTGTA-3' } \\
\text { Reverse: 5'-AGAAATCTCGAAGGCCTGGT-3' }\end{array}$ \\
\hline \multirow[t]{2}{*}{ CPT1 } & Forward: 5'- AGCAACTAAACAACCTGCCAGTACTA-3 \\
\hline & Reverse: 5'-GTCCGGATATTCAAGGATGCA-3' \\
\hline \multirow[t]{2}{*}{ PPAR-a } & Forward: 5'-CCTCAGGGTACCACTACGGAGT-3' \\
\hline & Reverse: 5'-GCCGAATAGTTCGCCGAA-3' \\
\hline \multirow[t]{2}{*}{ PPAR- $\gamma$} & Forward: 5'-AGGCCGAGAAGGAGAAGCTGTTG-3' \\
\hline & Reverse: 5'-TGGCCACCTCTTTGCTGTGCTC-3' \\
\hline \multirow[t]{2}{*}{$C / E B P-a$} & Forward: 5'-TGGACAAGAACAGCAACGAGTAC-3' \\
\hline & Reverse: 5'-GCAGTTGCCCATGGCCTTGAC-3' \\
\hline \multirow[t]{2}{*}{ GAPDH } & Forward: 5'-ACCCAGAAGACTGTGGATGG-3' \\
\hline & Reverse: 5'-ACACATTGGGGGTAGGAACA-3' \\
\hline \multirow[t]{2}{*}{ CYPTA1 } & Forward: 5'-AGCAACTAAACAACCTGCCAGTACTA-3' \\
\hline & Reverse: 5'-GTCCGGATATTCAAGGATGCA-3' \\
\hline
\end{tabular}

LPL lipoprotein lipase, CPT1 carnitine palmitoyltransferase 1, PPAR-a peroxisome proliferator-activated receptor alpha, $P P A R-\gamma$ peroxisome proliferator-activated receptor gamma, C/EBP- $a$ CCAAT/enhances binding protein alpha, CYP7A1 cholesterol 7 alpha hydroxylase, GADPH glyceraldehyde-3-phosphate dehydrogenase

analysis of variance was and a Tukey's multiple comparisons test were used for between-group comparisons. $\mathrm{P}<0.05$ was considered statistically significant.

\section{Results}

\section{Compositional analysis of the lotus leaf extract}

Figure 1 shows the activated chemical compounds in the lotus leaf extract via HPLC. Based on the data, the corresponding regression equations were established, with the content of standards as abscissa and the peak area as the ordinate (Table 2). Components in lotus leaf extract were calculated by peak area.

\section{Effect of the lotus leaf extract on mouse body weight}

At the beginning of the experiment, there was no difference in mouse body weight between the groups $(P>0.05)$. After feeding a high-fat diet for 7 weeks, the mouse weights in the model group increased faster than those of the normal group (Fig. 2). After intervention, the mice in treatment group gained weight slower than did the model group $(\mathrm{P}<0.05)$. And no significant difference was observed in LL-H group and L-carnitine group $(\mathrm{P}>0.05)$.

\section{Liver and epididymal fat indexes}

Obesity is often accompanied by an increase of white fat, which is manifested by the weight increase of white adipose tissue, and epididymal fat tissue is typical white adipose tissue. After the mice were sacrificed, their epididymal white adipose tissue is weighed and compared with body weight to obtain epididymal fat indexes, 


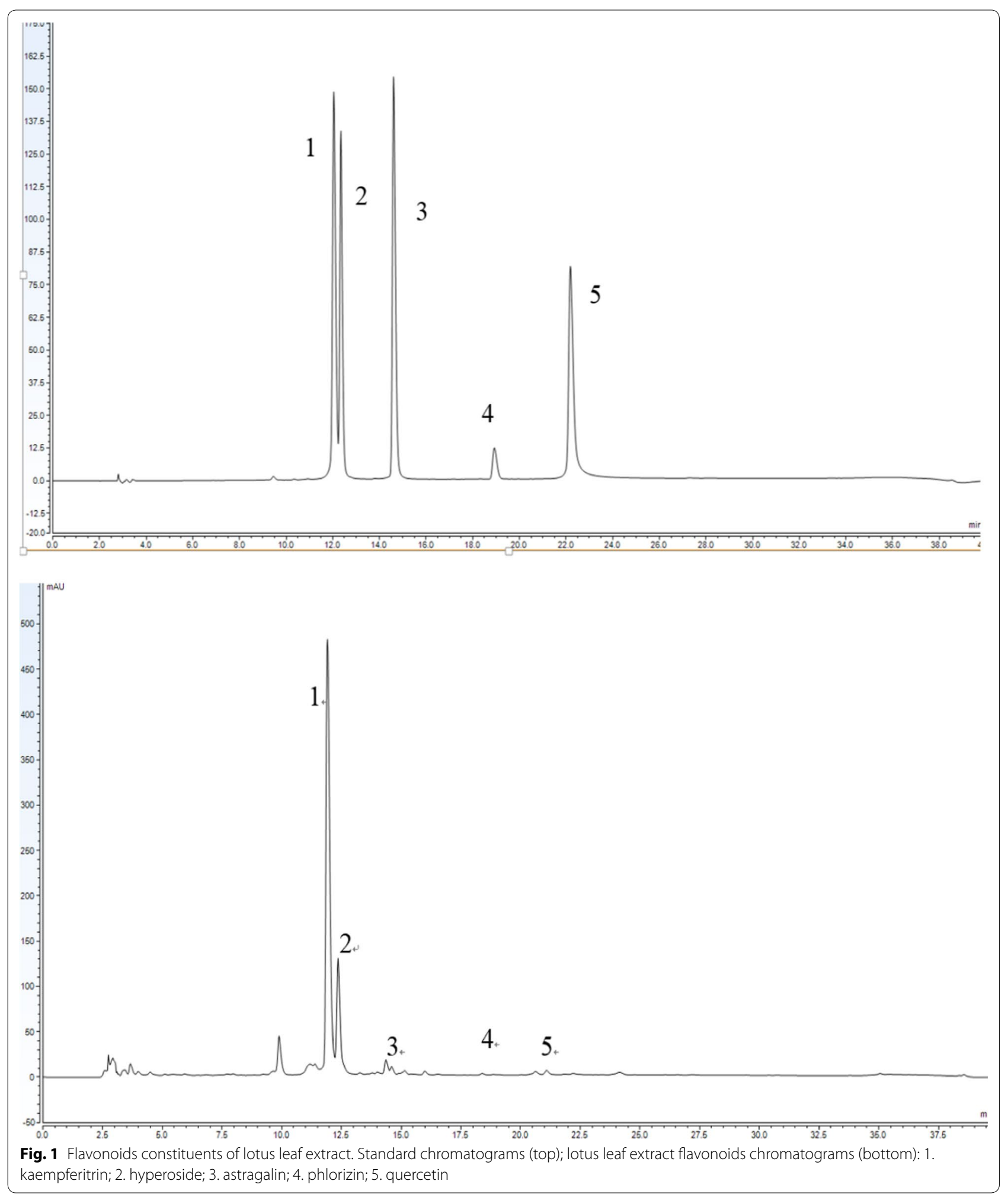

which could be used to explain the degree of obesity in mice. Obesity is often accompanied by disorder of lipid metabolism in the liver, which manifests as liver enlargement due to lipid accumulation, leading to an increase in liver weight.

The liver and epididymal fat indexes of the model group were highest, indicating fat accumulation in the liver and 
Table 2 Flavonoid content in lotus leaf extract

\begin{tabular}{llll}
\hline Compound & Regression equation & $\mathbf{R}^{\mathbf{2}}$ & $\begin{array}{l}\text { Content in lotus } \\
\text { leaf extract (mg/g) }\end{array}$ \\
\hline Kaempferitrin & $y=8.1347 x+3.7415$ & 0.9844 & $75.79 \pm 0.02$ \\
Hyperoside & $y=3.1862 x+9.5029$ & 0.9897 & $31.11 \pm 0.02$ \\
Astragalin & $y=11.338 x+0.8515$ & 0.9994 & $9.53 \pm 0.01$ \\
Phloretin & $y=0.9844 x+0.3039$ & 0.9843 & $0.26 \pm 0.01$ \\
Quercetin & $y=10.386 x-3.0995$ & 0.9990 & $0.096 \pm 0.05$ \\
\hline
\end{tabular}

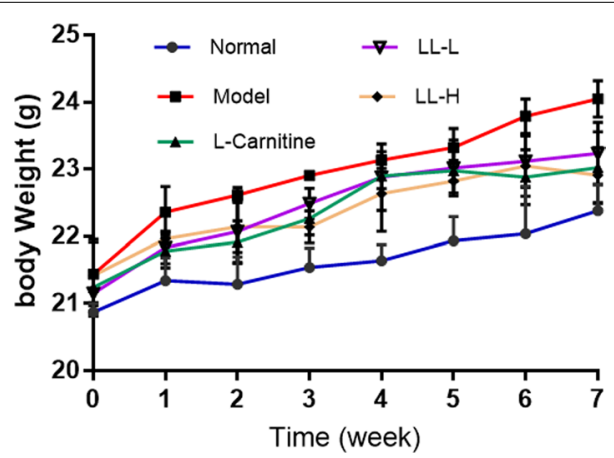

Fig. 2 Body weight gain. The data are shown as mean $\pm S D(n=10)$. L-Carnitine, mice treated with $200 \mathrm{mg} / \mathrm{kg}$ L-carnitine; LL-L, mice treated with $100 \mathrm{mg} / \mathrm{kg}$ lotus leaf extract; LL-H, mice treated with $200 \mathrm{mg} / \mathrm{kg}$ lotus leaf extract

epididymal tissues (Table 3). Compared with the model group, the liver and epididymal fat indexes of the L-carnitine and LL-H groups were reduced to different degrees $(\mathrm{P}<0.05)$.

\section{Histopathological examination of the liver and epididymal} fat

In the normal group, the hepatocytes were arranged neatly, with complete structure and fewer fat vacuoles (Fig. 3a). Hepatocytes in the model group were arranged irregularly with many fat vacuoles of different sizes. The hepatic cords radiating around the central vein were invisible. In the L-carnitine and LL groups after treatment, the radial hepatic cords around the central vein were clearly visible, few fat vacuoles were observed, and the hepatocytes were neatly arranged and structurally intact.

In the normal group, the adipocytes in the epididymal fat tissues were smallest, with a uniform shape (Fig. 3b). In the model group, the adipocytes were largest and uneven. The epididymal adipocytes were smaller in the L-carnitine and LL groups than in the model group, indicating effectively less fat cell hypertrophy.

\section{Serum levels of the biochemical indicators ALT, AST and AKP in mice}

The ALT, AST, and AKP levels in the model mouse group were significantly higher than those in the normal group ( $\mathrm{P}<0.05$; Fig. 4), indicating that obesity caused liver damage. After treatment, the serum ALT, AST, and AKP levels were significantly decreased compared with those of the control group $(\mathrm{P}<0.05)$. The high-concentration lotus leaf extract was more effective than was the low-concentration extract.

\section{Serum levels of TC, TG, LDL-C and HDL-C in mice}

The serum TC, TG and LDL-C levels were increased in the high-fat-diet-induced hyperlipidemic mice, but the HDL-C level was decreased compared with that in the normal mice $(\mathrm{P}<0.05$; Fig. 5). The lotus leaf extract decreased the TC, TG and LDL-C levels and increased the HDL-C level in the hyperlipidemic mice, and the results for both the high-concentration lotus leaf extract and L-carnitine groups were comparable.

\section{Serum levels of TNF- $\alpha$, IFN- $\gamma$, IL- $1 \beta$, IL- 6 , IL-4, and IL-10 in mice}

In all experimental groups, TNF- $\alpha$, IFN- $\gamma$, IL- $1 \beta$, and IL- 6 levels were highest, and the IL-4 and IL-10 levels were lowest in the model mice (Fig. 6). After intervention, serum TNF- $\alpha$, IFN- $\gamma$, IL- $1 \beta$, and IL- 6 contents were decreased in the LL-L, LL-H, and L-carnitine groups, IL-4 and IL-10 was increased compared with that in the model group $(\mathrm{P}<0.05)$.

\section{PPAR- $\gamma, C / E B P-a, P P A R-a, L P L, C P T 1$ and CYP7A1 mRNA expressions in the liver tissue}

To investigate the changes in gene expressions regulated by lotus leaf extracts in mice, we analyzed the lipid

Table 3 Liver and epididymal fat indexes of mice in each group

\begin{tabular}{lllll}
\hline Group & Normal & Model & L-Carnitine & LL-L \\
\hline Liver index & $3.81 \pm 0.30^{c}$ & $4.17 \pm 0.22^{\mathrm{a}}$ & $3.86 \pm 0.19^{\mathrm{b}}$ & $4.02 \pm 0.16^{\mathrm{ab}}$ \\
Epididymal fat index & $1.01 \pm 0.23^{\mathrm{b}}$ & $1.55 \pm 0.28^{\mathrm{a}}$ & $1.02 \pm 0.14^{\mathrm{b}}$ & $3.93 \pm 0.21^{\mathrm{bc}}$ \\
\hline
\end{tabular}

Values presented are the mean \pm standard deviation (liver Index: $n=10 /$ group, epididymal fat index: $n=5 / g r o u p$ )

a-c Mean values with different superscript letters are significantly different $(P<0.05)$ according to Tukey's multiple comparisons test. $L-c a r n i t i n e$, mice treated with $200 \mathrm{mg} / \mathrm{kg}$ L-carnitine; LL-L, mice treated with $100 \mathrm{mg} / \mathrm{kg}$ lotus leaf extract; LL-H, mice treated with $200 \mathrm{mg} / \mathrm{kg}$ lotus leaf extract 


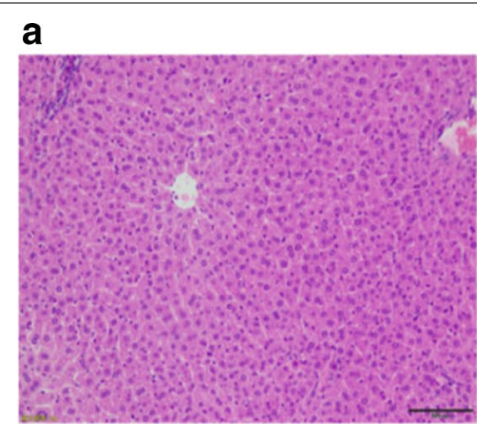

Normal

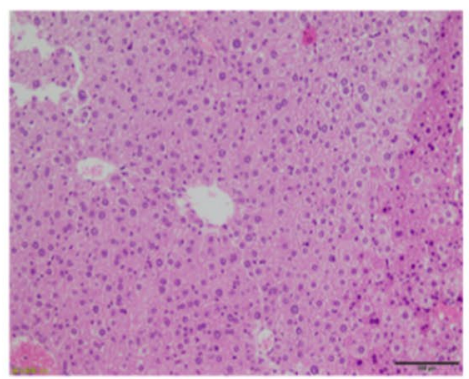

Model

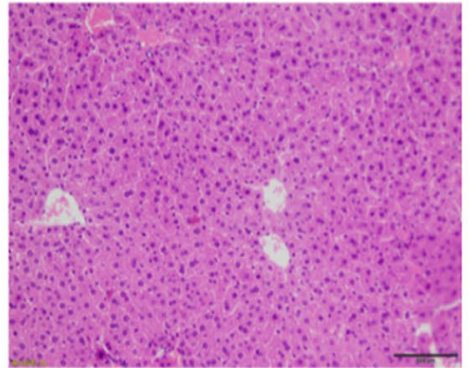

L-Carnitine

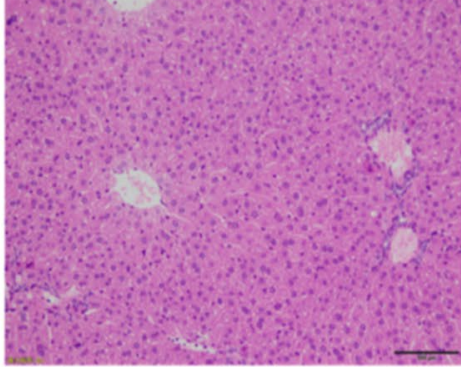

LL-H

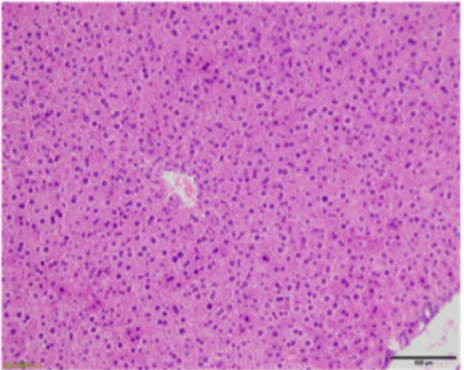

LL-L

b

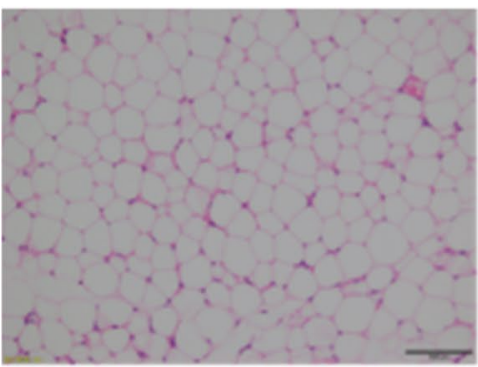

Normal

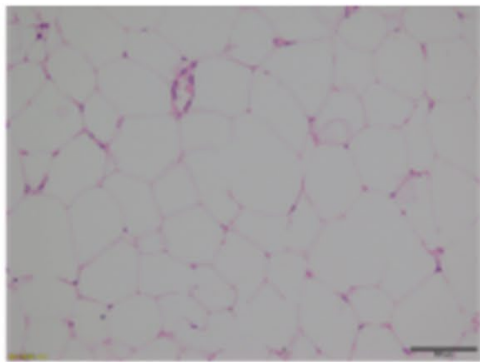

Model

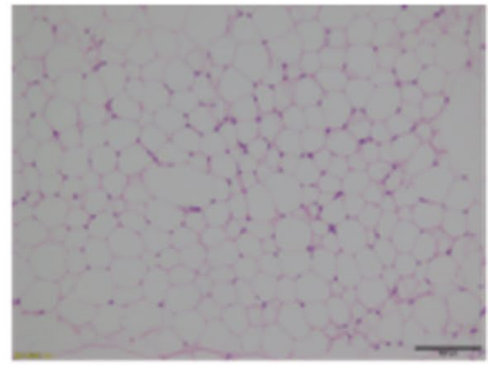

L-Carnitine

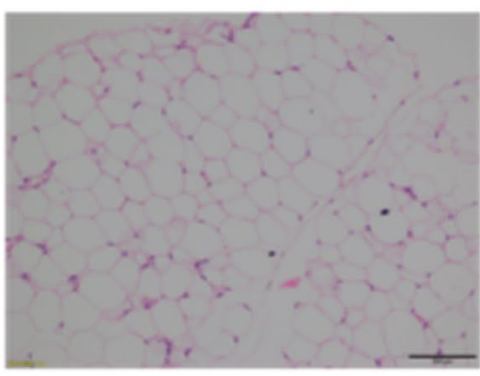

LL-H

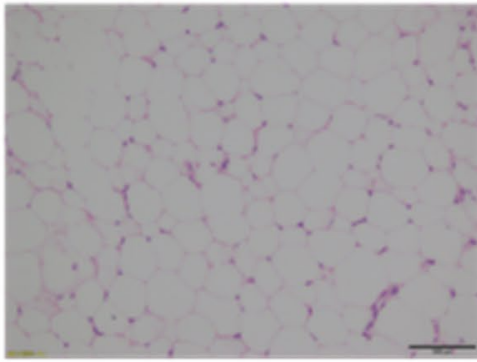

LL-L

Fig. 3 a Histopathological observation of liver sections in mice of the different groups after staining with hematoxylin and eosin (H\&E). $\mathbf{b} H \& E$ histopathological observation of the epididymal fat tissue in mice of the different groups

metabolism-related genes (Fig. 7). PPAR- $\alpha, L P L, C P T 1$, and CYP7A1 mRNA expression levels were lowest in the livers of the model mice, and PPAR- $\gamma$ and $C / E B P-\alpha$ expressions were highest. These results were opposite those of the normal group $(\mathrm{P}<0.05)$. lotus leaf extract and L-carnitine suppressed the PPAR- $\gamma$ and $C / E B P-\alpha$ mRNA 

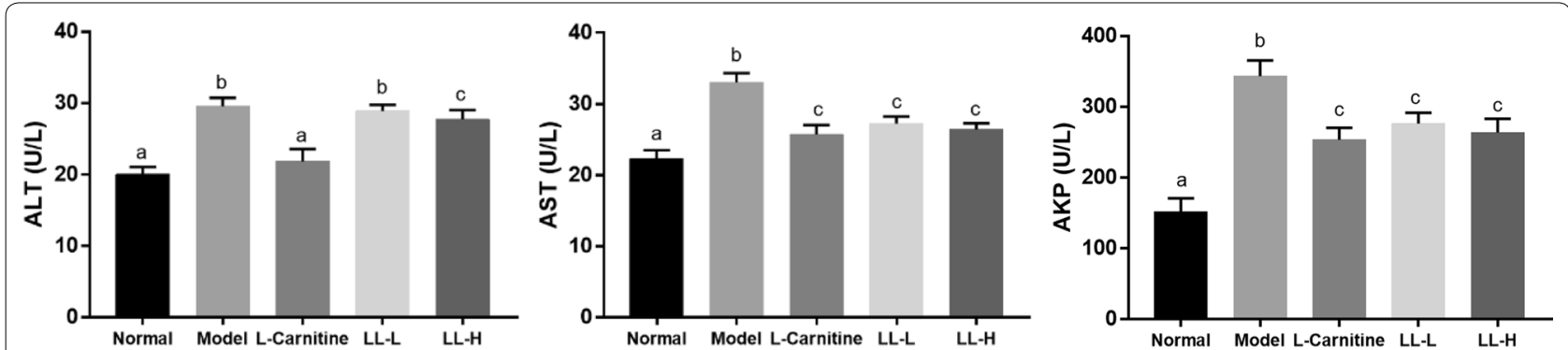

Fig. 4 Serum levels of ALT, AST, and AKP in mice of the different groups. The data are shown as mean $\pm S D(n=10) .{ }^{a-c}$ Mean values with different letters are significant difference $(P<0.05)$ according to according to Tukey's multiple comparisons test
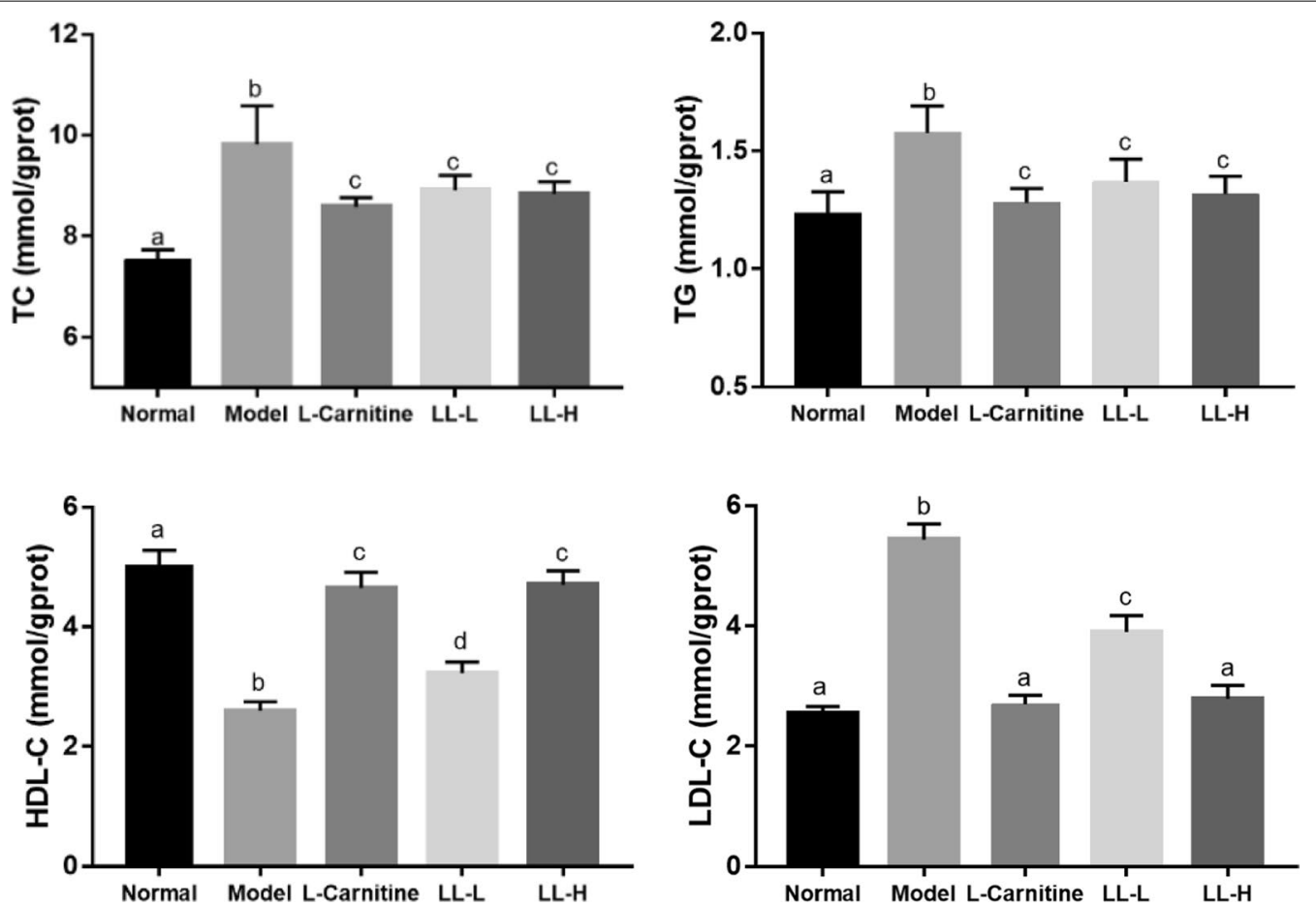

Fig. 5 Serum levels of TC, TG, HDL-C, and LDL-C in mice of the different groups. The data are shown as mean $\pm S D(n=10)$. ${ }^{a-d}$ Mean values with different letters are significant difference $(P<0.05)$ according to according to Tukey's multiple comparisons test

expressions and enhanced the PPAR- $\alpha, L P L, C P T 1$, and CYP7A1 mRNA expressions. The high-concentration lotus leaf extract had a more pronounced effect.

\section{Discussion}

High-energy diets and low-exercise lifestyles are becoming increasingly common; thus, the incidence of obesity increases annually. Long-term high-fat diets are the main cause of lipid metabolism disorders. Compared with healthy people, those with dyslipidemia present marked increases in TG, TC, LDL-C and free fatty acid levels as well as decreased HDL-C [27]. As the main organ for lipid metabolism, the liver is involved in $\beta$-oxidation of fatty acids, adipogenesis, and lipoprotein uptake and secretion, playing an important role in lipid metabolism [28]. In obese people, dyslipidemia leads to fat accumulation in hepatocytes, followed by the entire liver, with the result of enlarged due to lipid accumulation and a certain increase in liver weight. The fat vacuole areas and fat infiltration are increased, that further cause liver injury $[29,30]$. Obesity is significantly positively correlated with various abnormalities in liver function indicators, such as the serum enzyme indicators ALT, AST, and AKP, which are significantly increased [31]. Also, the obesity is often 

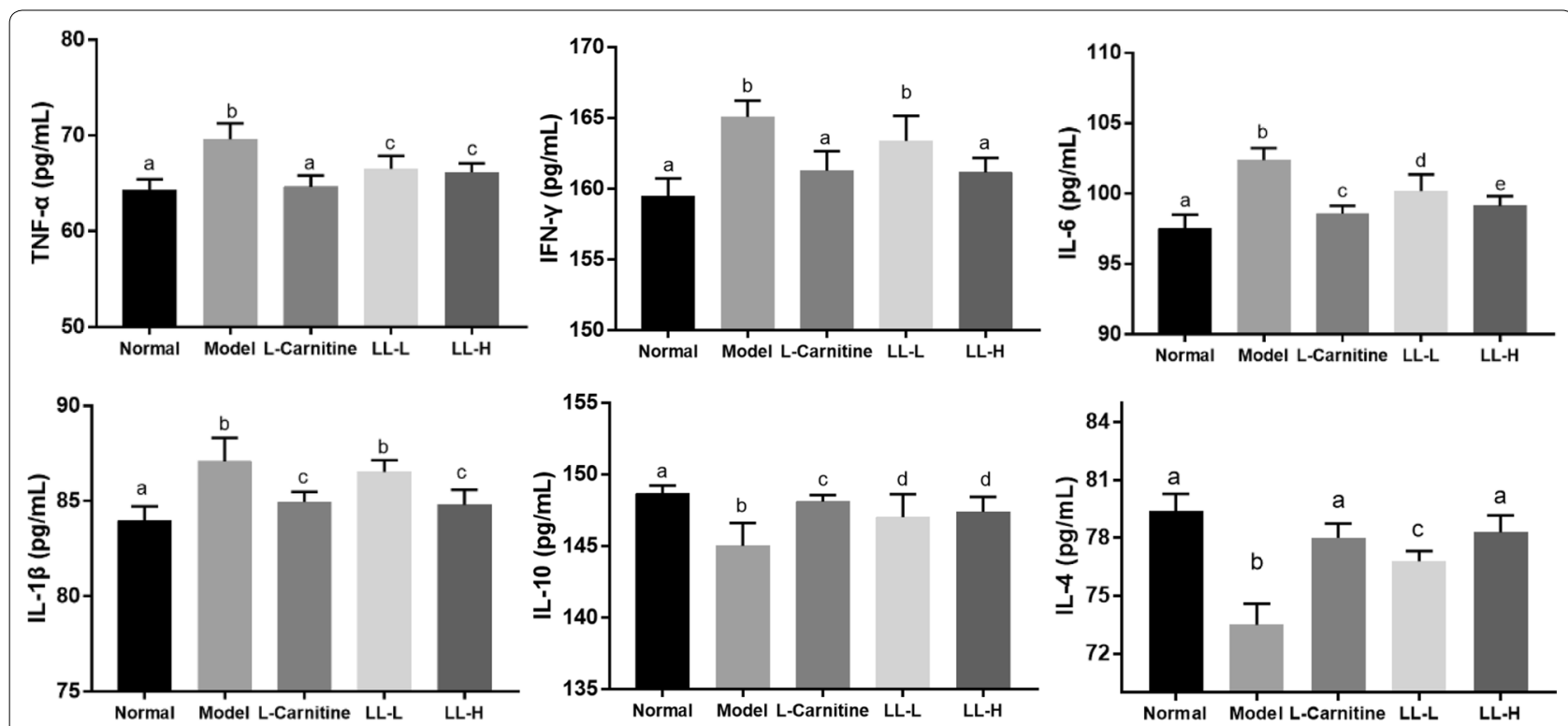

Fig. 6 Serum levels of the cytokines TNF- $a$, IFN- $\gamma$, IL-1 $\beta, I L-6, I L-4$, and IL-10 in mice of the different groups. The data are shown as mean \pm SD $(n=10) .{ }^{a-e}$ Mean values with different letters are significant difference $(P<0.05)$ according to according to Tukey's multiple comparisons test
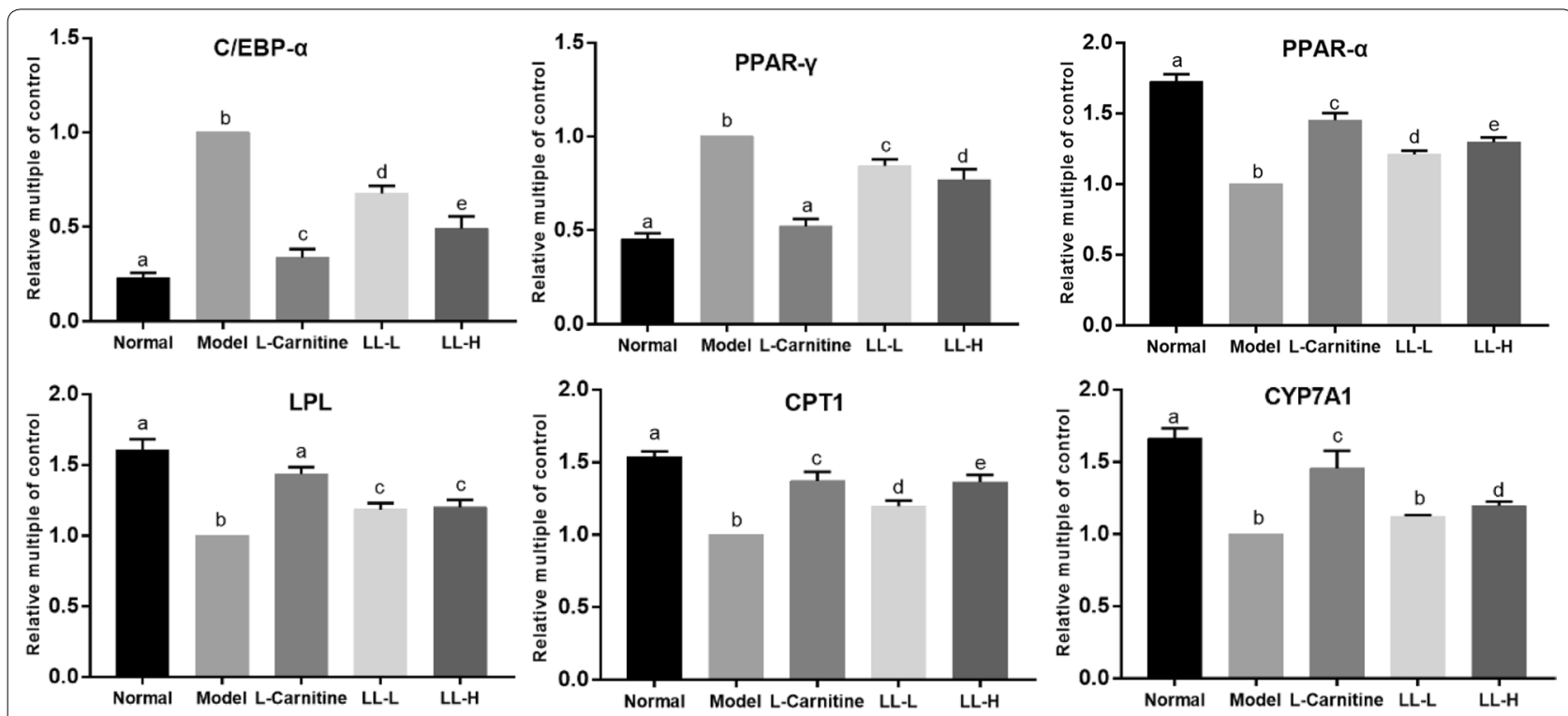

Fig. 7 mRNA expression levels of PPAR- $\gamma, C / E B P-a, P P A R-a, L P L, C P T 1$, and CYP7A1 in liver tissues of the different groups were investigated by RT-qPCR. The data are shown as mean $\pm S D(n=10) .{ }^{a-e}$ Mean values with different letters are significant difference $(P<0.05)$ according to according to Tukey's multiple comparisons test

accompanied by an increase in the weight of white fat tissue, and epididymal fat tissue is typical of white fat tissue. Lotus leaf extract inhibited the increased body weight, liver quality, and epididymal fat mass, reduced the TG, TC, LDL-C levels, and significantly increased the HDL-C levels in obese mice. The extract also alleviated hepatic fat vacuoles and excessive fat accumulation and decreased the serum ALT, AST, and AKP levels to prevent early liver injury.

Obesity is a state of systemic chronic low-grade inflammation [32], mainly resulting from the interaction between adipose tissue and macrophages. In addition to large amounts of free fatty acids, adipose tissue also secretes TNF- $\alpha$, IL-6, IL-1, and the macrophage 
migration inhibitor, monocyte chemoattractant protein-1, which causes an imbalance between the proinflammatory and anti-inflammatory cytokines, leading to chronic persistent inflammation [33, 34]. Lotus leaf extracts significantly inhibit the release of inflammatory cytokines IL- $1 \beta$, IL- 6 , TNF- $\alpha$, and IFN- $\gamma$ and promote the release of anti-inflammatory IL- 4 and IL-10 to alleviate chronic low-level inflammation accompanied by obesity.

When preadipocytes differentiate into adipocytes, transcription factors regulate adipocyte formation by regulating the expression of differentiation-related proteins. PPAR- $\gamma[35,36]$ and C/EBP- $\alpha[37]$ are core transcription factors involved in adipocyte differentiation, and positive feedback occurs between them to induce differentiation, cloning and proliferation of adipocytes [38]. The PPAR family regulates lipid metabolism and participates in energy metabolism and lipid deposition by regulating the transcription of multiple target genes. PPAR- $\alpha$ is a subtype of PPAR and plays an important role in lipid metabolism in the liver [39]. PPAR- $\alpha$ regulates the expression of genes related to lipid and lipoprotein metabolism, which promotes $\beta$-oxidation of fatty acids, ketogenesis, metabolism and TG storage and reduces lipid accumulation, thereby modulating lipid metabolism disorders [40]. PPAR- $\gamma$ plays the opposite role by promoting lipid synthesis and fat accumulation by regulating lipid-related genes.

CPT-1 is a rate-limiting enzyme for fatty acid oxidation and promotes $\beta$-oxidation of fatty acids [41]. Lipoprotein lipase (LPL) catalyzes the decomposition of TG from chylomicron (CM) and very low-density lipoprotein (VLDL) into fatty acids and monoglycerides for tissue oxidation and energy storage [42]. LPL also participates in converting apolipoproteins and phospholipids between VLDL and HDL, thus increasing HDL-C levels [42]. These two lipases are target genes regulated by PPAR- $\alpha$ and PPAR- $\gamma$ [43-45]. CYP7A1 is a rate-limiting enzyme that catalyzes bile acid synthesis by cholesterol, which is important for maintaining the internal balance of bile acids [46]. Lotus leaf extracts reduce adipocyte differentiation by downregulating PPAR $-\gamma$ and $C / E B P-\alpha$ mRNA expressions and upregulating PPAR- $\alpha$, CPT- 1, LPL and CYP7A1 mRNA expressions, thus reducing fat accumulation and accelerating fat oxidation to modulate lipid metabolism disorders.

In our experiment, flavonoid glycosides were the main compounds isolated from lotus leaf, which had good physiological activities. Kaempferitrin could down-regulate the mRNA expression of lipid synthesis genes and pro-inflammatory genes TLR4, TNF- $\alpha$, NLRP3, Caspase-1, IL-18, IL-1 $\beta$, SRRBP-1c, PPAR- $\gamma$, and elevate
PPAR- $\alpha$ mRNA expression in obese Wistar rats [47]. In human RA-FLS MH7A cell line test, kaempferitrin exerted anti-inflammatory activity by reducing IL- $1 \beta$, IL-6, TNF- $\alpha$, matrix metalloproteinase (MMP)-1 and MMP-3 levels [48]. Hyperoside, which has good antioxidant capacity, protected the liver from damage by increasing antioxidant enzyme activities, inhibiting oxidative stress and lipid peroxidation damage $[49,50]$. And anti-inflammatory effect of hyperoside was achieved by inhibiting NF- $\mathrm{KB}$ (nuclear factor kappa-B) activation and IкB (inhibitor of NF- $\kappa B$ )- $\alpha$ degradation [51]. Astragalin have a good inhibition of lipogenesis and fat accumulation in 3T3-L1 adipocytes, which showed antiobesity effect by down-regulating the mRNA expression of PPAR- $\gamma$, C/EBP- $\alpha$, FAS, and leptin [52]. At the same time, other physiological activity studies have found that astragalin have the ability to improve anti-oxidant and anti-inflammatory activities [53, 54]. Flavonoids regulate signal transduction and transcription factor expression and inhibit oxidative stress to suppress inflammation [55]. Flavonoids in lotus leaf cooperated with each other to fight against obesity. Therefore, lotus leaf extracts regulated lipid-related genes, including $P P A R-\gamma, C / E B P-\alpha$, PPAR- $\alpha, L P L, C P T 1$, and CYP7A1, to reduce adipocyte differentiation and fat accumulation and promote oxidation of fat for energy supplies. Lotus leaves may prevent and treat obesity and have a high value in the medicinal and food industries.

\section{Acknowledgements}

Not applicable.

\section{Authors' contributions}

YW performed the majority of the experiments and wrote the manuscript; FT, TYZ, BLX and LXR contributed to the data analysis; XZ designed and supervised the study, and checked the final manuscript. All authors read and approved the final manuscript.

\section{Funding}

This research was supported by the Scientific Research Foundation for Returned Overseas Chinese Scholars, and the State Education Ministry [Jiaowaisiliu (2014)1685], China.

\section{Availability of data and materials}

Datasets used and/or analysed during the current study that are not included in the manuscript are available from the corresponding author on reasonable request.

\section{Competing interests}

The authors declare that they have no competing interests.

\section{Author details \\ ${ }^{1}$ Chongqing Collaborative Innovation Center for Functional Food, Chongqing University of Education, Chongqing 400067, China. ${ }^{2}$ Chongqing Engineer- ing Research Center of Functional Food, Chongqing University of Education, Chongqing 400067, China. ${ }^{3}$ Chongqing Engineering Laboratory for Research and Development of Functional Food, Chongqing University of Education, Chongqing 400067, China. ${ }^{4}$ College of Biological and Chemical Engineering, Chongqing University of Education, Chongqing 400067, China. ${ }^{5}$ Department of Public Health, Our Lady of Fatima University, Valenzuela 838, Philippines.}


Received: 13 July 2020 Accepted: 8 September 2020

Published online: 25 September 2020

\section{References}

1. Thomas EL, Frost G, Taylor-Robinson SD, Bell JD (2012) Excess body fat in obese and normal-weight subjects. Nutr Res Rev 25:150-161

2. Liu D, Hao YX, Zhao TZ (2019) Childhood BMI and adult obesity in a Chinese sample: a13-year follow-up study. Biomed Environ Sci 32:162-168

3. Younossi ZM, Koenig AB, Abdelatif D, Fazel Y, Henry L, Wymer M (2016) Global epidemiology of nonalcoholic fatty liver disease-Meta-analytic assessment of prevalence, incidence, and outcomes. Hepatology 64:73-84

4. Landsberg L, Aronne LJ, Beilin L, Burke V, Igel LI, Lloyd-Jones D, Sowers J (2013) Obesity-related hypertension: pathogenesis, cardiovascular risk, and treatment: a position paper of The Obesity Society and the American Society of Hypertension. J Clin Hypertens 15:14-33

5. Khaodhiar L, Cummings S, Apovian CM (2009) Treating diabetes and prediabetes by focusing on obesity management. Curr Diab Rep 9:348-354

6. Khandekar MJ, Cohen P, Spiegelman BM (2011) Molecular mechanisms of cancer development in obesity. Nat Rev Cancer 11:886-895

7. Chen IJ, Liu CY, Chiu JP, Hsu CH (2016) Therapeutic effect of high-dose green tea extract on weight reduction: a randomized, double-blind, placebo-controlled clinical trial. Clin Nutr 35:592-599

8. National pharmacopoeia committee (2000) Pharmacopoeia of the People's Republic of China. Chemical industry press, Beijing, pp 262-263

9. Grant WF, Sidhu BS (1967) Basic chromosome number, cyanogenetic glucoside variation, and geographic distribution of lotus species. Can J Bot 45:639-647

10. Chen X, Qi J (2015) Advances in the study of flavonoids and alkaloids in lotus leaves. Chin J Exp Tradit Med Formulae 21:211-214

11. Ding YB, Pu LN, Kan JQ (2017) Hypolipidemic effects of lipid-lowering granulated tea preparation from monascus-fermented grains (adlay and barley bran) mixed with lotus leaves on sprague-dawley rats fed a highfat diet. J Funct Foods 32:80-89

12. Huang B, Ban XQ, He JS, Tong J, Tian J, Wang YW (2010) Hepatoprotective and antioxidant activity of ethanolic extracts of edible lotus (Nelumbo nucifera Gaertn.) leaves. Food Chem 120:873-878

13. Subashini R, Rajadurai M (2011) Evaluation of cardioprotective efficacy of Nelumbo Nucifera leaf extracton isoproterenol-induced myocardial infarction in wistar rats. Int J Pharma Bio Sci 2:285-294

14. Nakamura S, Nakashima S, Tanabe G (2013) Alkaloid constituents from flower buds and leaves of sacred lotus (Nelumbo nucifera) with melanogenesis inhibitory activity in B16 melanoma cells. Bioorg Med Chem 21:779-787

15. Ahn JH, Kim ES, Lee C, Kim S, Cho SH, Hwang BY, Lee MK (2013) Chemical constituents from Nelumbo nucifera leaves and their anti-obesity effects. Bioorg Med Chem Lett 23:3604-3608

16. Ma C, Li G, He Y, Xu B, Mi X, Wang H, Wang Z (2015) Pronuciferine and nuciferine inhibit lipogenesis in 3T3-L1 adipocytes by activating the AMPK signaling pathway. Life Sci 136:120-125

17. Ma C, Wang J, Chu H, Zhang X, Wang Z, Wang H, Li G (2014) Purification and characterization of aporphine alkaloids from leaves of Nelumbo nucifera Gaertn and their effects on glucose consumption in 3T3-L1 adipocytes. Int J Mol Sci 15:3481-3494

18. Siegner R, Heuser S, Holtzmann U, Söhle J, Schepky A, Raschke T, Stäb F, Wenck H, Winnefeld M (2010) Lotus leaf extract and L-carnitine influence different processes during the adipocyte life cycle. Nutr Metab 7:66

19. Ono Y, Hattori E, Fukaya Y, Imai S, Ohizumi Y (2006) Anti-obesity effect of Nelumbo nucifera leaves extract in mice and rats. J Ethnopharmaco 106:238-244

20. Du H, You J, Zhao X, Park JY, Kim SH, Chang KJ (2010) Antiobesity and hypolipidemic effects of lotus leaf hot water extract with taurine supplementation in rats fed a high fat diet. J Biomed Sci 17:S42

21. Chen GL, Zhu MZ, Guo MQ (2019) Research advances in traditional and modern use of Nelumbo nucifera: phytochemicals, health promoting activities and beyond. Crit Rev Food Sci 59:S189-S209

22. Bastard JP, Maachi M, Lagathu C, Kim MJ, Caron M, Vidal H, Capeau J, Feve $B$ (2006) Recent advances in the relationship between obesity, inflammation, and insulin resistance. Eur Cytokine Netw 17:4-12
23. Kim BM, Cho BO, Jang SI (2019) Anti-obesity effects of Diospyros lotus leaf extract in mice with high-fat diet-induced obesity. Int J Mol Med 43:603-613

24. Wu CH, Yang MY, Chan KC, Chung PJ, Ou TT, Wang CJ (2010) Improvement in high-fat diet-induced obesity and body fat accumulation by a Nelumbo nucifera leaf flavonoid-rich extract in mice. J Agric Food Chem 58:7075-7081

25. Zhang L, Li MX, Wu XL, Qiao XG (2014) Study on separation and purification of total flavones from lotus leaf by $A B-8$ macroporous resin. Food Res Dev 10:42-46

26. Hasty AH, Gruen ML, Terry ES, Surmi BK, Atkinson RD, Gao L, Morrow JD (2007) Effects of vitamin E on oxidative stress and atherosclerosis in an obese hyperlipidemic mouse model. J Nutr Biochem 18:127-133

27. Smith CJ, Ryckman KK (2015) Epigenetic and developmental influences on the risk of obesity, diabetes, and metabolic syndrome. Diabetes Metab Syndr Obes 8:295-302

28. Rottiers V, Näär AM (2012) Micro RNAs in metabolism and metabolic disorders. Nat Rev Mol Cell Biol 13:239-250

29. Gariani K, Ryu D, Menzies KJ, Yi HS, Stein S, Zhang H, Perino A, Lemos V, Katsyuba E, Jha P, Vijgen S, Rubbia-Brandt L, Kim YK, Kim JT, Kim KS, Shong M, Schoonjans K, Auwerx J (2017) Inhibiting poly ADP-ribosylation increases fatty acid oxidation and protects against fatty liver disease. J Hepatol 66:132-141

30. Xiao CT, Hsieh J, Adeli K, Lewis GF (2011) Gut-liver interaction in triglyceride-rich lipoprotein metabolism. Am J Physiol Endocrinol Metab 301:E429-E446

31. Wong D, Bach H, Sun J, Hmama Z, Av-Gay Y (2011) Mycobacterium tuberculosis protein tyrosine phosphatase (PtpA) excludes host vacuolarH+-ATPase to inhibit phagosome acidification. Proc Natl Acad Sci USA 108:19371-19376

32. Hotamisligil GS, Gokhan S (2006) Inflammation and metabolic disorders. Nature 444:860-867

33. Hummasti S, Hotamisligil GS (2010) Endoplasmic reticulum stress and inflammation in obesity and diabetes. Circ Res 107:579-591

34. Hotamisligil GS (2017) Inflammation, metaflammation and immunometabolic disorders. Nature 542:177-185

35. Mueller E, Drori S, Aiyer A, Yie J, Sarraf P, Chen H, Hauser S, Rosen ED, Ge K, Roeder RG, Spiegelman BM (2002) Genetic analysis of adipogenesis through peroxisome proliferator-activated receptor gamma isoforms. J Biol Chem 2277:41925-41930

36. Xu LY, Ma XR, Verma NK, Wang DM, Gavrilova O, Proia RL, Finkel T, Mueller E (2018) Ablation of PPARy in subcutaneous fat exacerbates age-associated obesity and metabolic decline. Aging Cell 17:e1272

37. Liu HR, Xiong YQ, Zhu XF, Gao H, Yin SJ, Wang JF, Chen GM, Wang CP, Xiang L, Wang PP, Fang J, Zhang RH, Yang L (2017) Icariin improves osteoporosis, inhibits the expression of PPARy, C/EBPa, FABP4 mRNA, N1ICD and jagged 1 proteins, and increases Notch2 mRNA in ovariectomized rats. Exp Ther Med 13:1360-1368

38. Balakrishnan BB, Krishnasamy K, Choi KC (2018) Moringa concanensis Nimmo ameliorates hyperglycemia in 3T3-L1 adipocytes by upregulating PPAR- $\gamma$, C/EBP-a via Akt signaling pathway and STZ-induced diabetic rats. Biomed Pharmacother 103:719-728

39. Gross B, Pawlak M, Lefebvre P, Staels B (2017) PPARs in obesity-induced T2DM, dyslipidaemia and NAFLD. Nat Rev Endocrinol 13:36-49

40. Ma XR, Xu LY, Wang S, Cui B, Li XY, Xu JM, Ning G (2011) Deletion of steroid receptor coactivator-3 gene ameliorates hepatic steatosis. J Hepato 55:445-452

41. Chen LY, Yang BS, Zhou L, Ren F, Duan ZP, Ma YJ (2015) Promotion of mitochondrial energy metabolism during hepatocyte apoptosis in a rat model of acute liver failure. Mol Med Rep 12:5035-5041

42. Cruciani-Guglielmacci C, Magnan C (2017) Brain lipoprotein lipase as a regulator of energy balance. Biochimie 143:51-55

43. Yang H, Suh DH, Kim ED (2018) Metabolomic and lipidomic analysis of the effect of pioglitazone on hepatic steatosis in a rat model of obese type 2 diabetes. Br J Pharmacol 175:3610-3625

44. Ashish S, Rader DJ, Millar JS (2010) The effect of PPAR-alpha agonism on apolipoprotein metabolism in humans. Atherosclerosis 210:35-40

45. Niu YM, Yuan H, Fu L (2010) Aerobic exercise's reversal of insulin resistance by activating AMPKa-ACC-CPT1 signaling in the skeletal muscle of C57BL/6 mice. Int J Sport Nutr Exerc Metab 20:370-380 
46. Donepudi AC, Ferrell JM, Boehme S, Choi HS, Chiang JYL (2017) Deficiency of cholesterol 7a-hydroxylase in bile acid synthesis exacerbates alcohol-induced liver injury in mice. Hepatol Commun 2:99-112

47. Real-Sandoval SA, Gutiérrez-López GF, Domínguez-López A, PaniaguaCastro N, Michicotl-Meneses MM, Jaramillo-Flores ME (2020) Downregulation of proinflammatory liver gene expression by Justicia spicigera and kaempferitrin in a murine model of obesity-induced by a high-fat diet. J Funct Foods 65:103781

48. Wang J, Zhao Q (2019) Kaempferitrin inhibits proliferation, induces apoptosis, and ameliorates inflammation in human rheumatoid arthritis fibroblast-like synoviocytes. Phytother Res 33:1726-1735

49. Tang M, Liu Y, Xia PY (2011) Protective effect of hyperfine on acute liver injury in rats induced by $\mathrm{CCl}_{4}$. China Pharm 22:582-583

50. Choi JH, Kim DW, Yun N, Choi JS, Islam MN, Kim YS, Lee SM (2011) Protective effects of hyperoside against carbon tetrachloride-induced liver damage in mice. J Nat Prod 74:1055-1060

51. Kim SJ, Um JY, Lee JY (2011) Anti-inflammatory activity of hyperoside through the suppression of nuclear factor-kB activation in mouse peritoneal macrophages. Am J Chin Med 39:171-181

52. Muni Swamy G, Ramesh G, Prasad RD, Meriga B (2020) Astragalin, (3-O-glucoside of kaempferol), isolated from Moringa oleifera leaves modulates leptin, adiponectin secretion and inhibits adipogenesis in 3T3-L1 adipocytes. Arch Physiol Biochem. https://doi.org/10.1080/13813 455.2020 .1740742

53. Jia Q, Wang T, Wang X, Xu H, Liu Y, Wang Y, Shi Q, Liang Q (2019) Astragalin suppresses inflammatory responses and bone destruction in mice with collagen-induced arthritis and in human fibroblast-like synoviocytes. Front Pharmacol 10:94

54. Chen X, Cheng C, Zuo XZ, Huang W (2020) Astragalin alleviates cerebral ischemia-reperfusion injury by improving anti-oxidant and anti-inflammatory activities and inhibiting apoptosis pathway in rats. BMC Complement Med Ther 20:120

55. Hossain MK, Dayem AA, Han J, Yin YF, Kim K, Saha SK, Yang GM, Choi HY, Cho SG (2016) Molecular mechanisms of the anti-obesity and antidiabetic properties of flavonoids. Int J Mol Sci 7:569

\section{Publisher's Note}

Springer Nature remains neutral with regard to jurisdictional claims in published maps and institutional affiliations.

\section{Submit your manuscript to a SpringerOpen ${ }^{\circ}$ journal and benefit from:}

- Convenient online submission

- Rigorous peer review

- Open access: articles freely available online

- High visibility within the field

- Retaining the copyright to your article

Submit your next manuscript at $\boldsymbol{\nabla}$ springeropen.com 